\title{
Some Remarks on the Relative Cohen-Macaulay Property
}

\author{
Ibrahim Nonkané \\ Correspondence: Département d'économie et de mathématiques appliquées, IUFIC, Université Ouaga II, Burkina faso 12 \\ BP 417 Ouagadougou 12. E-mail: inonkane@univ-ouaga2.bf
}

Received: December 1, 2020 Accepted: January 12, 2021 Online Published: January 23, 2021

doi:10.5539/jmr.v13n1p41 URL: https://doi.org/10.5539/jmr.v13n1p41

\begin{abstract}
In this paper, we present a detailed proof of Knop's result on the relative Cohen-Macaulay property for invariant graded and finitely subalgebras. We announce the result for the case of formal power series, discuss the non-commutative version, and make interesting remarks.
\end{abstract}

Keywords: algebraic geometry, commutative algebra, Cohen-Macaulay property, homogeneous systems of parameters, invariant graded subalgebras

\section{Introduction}

The Cohen-Macaulay property is a classical subject named after Irvin Sol Cohen and Francis Sowerby Macaulay. CohenMacaulay rings and modules play role a central in modern commutative algebra. Let $R$ be a Noetherian local ring, and $M$ a finite $R$-module. If the algebraic invariant depth $M$ equals the geometric invariant $\operatorname{dim} M$, then $M$ is called a Cohen-Macaulay module. The ring $R$ is called Cohen-Macaulay ring if it is a Cohen-Macaulay module when viewed as a module over itself. In 1916 F. S. Macaulay proved the property for polynomial rings in finitely many indeterminates with coefficients in a field (Macaulay 1916) and in 1946 I.S.Cohen established the corresponding result for regular local rings (Cohen 1946) . While the notion of regularity has a very clear geometric interpretation that $t$ is the algebraic counterpart of the notion of a non-singular point, the geometric meaning of Cohen-Macaulay property is somewhat obscure, but the definition of Cohen-Macaulay property is sufficiently general to allow a wealth of examples in algebraic geometry, invariant theory, and combinatorics.

Many results have been obtained in this subject (Bruns \& Herzog 1998) but our interest goes the Hochster- Roberts theorem by which a ring of invariants of a linearly reductive group is Cohen-Macaulay (Hochster \& Roberts 1974) and the tight closure introduced by M. Hochster and C. Huneke ( Hochster \& Huneke 1990). In fact the ideas of the proof of results dealt in this paper come from the work of M. Hochster and C. Huneke ( Hochster \& Huneke 1990). This paper is partially expository in nature. The central result in this paper has been previously obtained by F. Knop (Knop 1991), but was never published. We found it somehow interesting and have taken the initiative to write down and expand it rigorously with every details in a very comprehensive way. In fact the result deals with the relative Cohen-Macaulay property for invariant graded and finitely generated subalgebras under some rational action. We analyze and deduce that the idea of the proof remains true for every finitely generated subalgebra, even for more general algebraic structure.

\subsection{Preliminaries}

In this subsection we recall some basics found in (Stanley 1986) that are useful in what follows. Let $k$ be a field, and let $R$ be an $\mathbb{N}^{m}$-graded connected commutative $k$-algebra with identity. Thus

$$
R=\coprod_{\alpha \in \mathbb{N}^{m}} R_{\alpha} \text { (vector space direct sum), } R_{\alpha} R_{\beta} \subset R_{\alpha+\beta}, R_{0}=k .
$$

Elements $x \in R_{\alpha}$ are said to be homogeneous of degree $\alpha$, denoted $\operatorname{deg} x=\alpha$. Let

$$
\mathcal{H}(R)=\bigcup_{\alpha} R_{\alpha},
$$

and

$$
R_{+}=\coprod_{\alpha \neq 0} R_{\alpha}
$$

The ideal $R_{+}$is the unique maximal homoegeneous ideal. 
The Krull dimension is defined by the following equal numbers:

$$
\begin{aligned}
\operatorname{dim} R:= & \text { maximum number of homogeneous elements of } R \\
& \text { algebraically independent over } k \\
:= & \text { length of longest chain of prime ideals of } R .
\end{aligned}
$$

Let $M$ be a $\mathbb{Z}^{m}$-graded $R$-module,

$$
\operatorname{dim} M:=\operatorname{dim}(R / \operatorname{Ann} M),
$$

where Ann $M:=\{r \in R \mid r M=0\}$ is the annihilator of the $R$-module $M$.

Definition 1.1. Let $R$ be an $\mathbb{N}^{m}$-graded $k$-algebra, and let $M$ be a $\mathbb{Z}^{m}$-graded $R$-module. A partial homogeneous system of parameters for $M$ is a sequence $\theta_{1}, \theta_{2}, \ldots, \theta_{r} \in \mathcal{H}\left(R_{+}\right)$such that $\operatorname{dim} M /\left(\theta_{1} M+\theta_{2} M+\cdots+\theta_{r} M\right)=\operatorname{dim} M-r$. An homogeneous system of parameters for $M$ is a partial homogeneous system of parameters for $M$ with $r=\operatorname{dim} M$. Equivalently, $\theta_{1}, \theta_{2}, \ldots, \theta_{d} \in \mathcal{H}\left(R_{+}\right)$is a homogeneous system of parameters for $M$ if only if $d=\operatorname{dim} M$ and $M$ is a finitely-generated $k\left[\theta_{1}, \theta_{2}, \ldots, \theta_{d}\right]$-module.

Definition 1.2. $\theta_{1}, \theta_{2}, \ldots, \theta_{r} \in \mathcal{H}\left(R_{+}\right)$is a homogeneous $M$-sequence (regular sequence) if $\theta_{i+1}$ is a non-zero-divisor on $M /\left(\theta_{1} M+\theta_{2} M+\cdots+\theta_{i} M\right), 0 \leq i<r$. Equivalently, $\theta_{1}, \theta_{2}, \ldots, \theta_{r}$ are algebraically independent over $k$ and $M$ is a free $k\left[\theta_{1}, \theta_{2}, \ldots, \theta_{d}\right]$-module.

An $M$-sequence is a partial homogeneous system of parameters for $M$ and if $m=1$ any two maximal $M$-sequences have the same length.

Definition 1.3. (1) If $m=1$, let depth $M:=$ length of longest homogeneous $M$-sequence.

(2) If $m>1$, specialize the grading to a $\mathbb{Z}$-grading in any way and define depth $M$ as in (1).

It is clear that depth $M \leq \operatorname{dim} M$. The case of equality, i.e, when some homogeneous system of parameters is regular, is of particular importance.

Definition 1.4. $M$ is Cohen-Macaulay if $\operatorname{depth} M=\operatorname{dim} M$.

Theorem 1.5. Let M have an homogeneous system of parameters. Then the following assertions are equivalent :

1. $M$ is Cohen-Macaulay;

2. every homogeneous system of parameters of $M$ is regular;

3. $M$ is finitely-generated and free $k\left[\theta_{1}, \theta_{2}, \ldots, \theta_{d}\right]$-module for some homogeneous system of parameters $\left(\theta_{1}, \theta_{2}, \ldots, \theta_{d}\right)$.

\section{The Relative Cohen-Macaulay Property}

Denote by $K$ a commutative field of characteristic zero. Let $n$ be a positive integer and consider the polynomial ring $K[x]$ in $n$ variables. Let $S$ be a graded and finitely generated subalgebra. Let $r+1$ be its dimension i.e. the dregree of transcendency ot its quotient field over $K$. Recall that a homogeneous system of parameters in $S$, consists of homogeneous polynomials $f_{0}, \ldots, f_{r}$ such that $S$ is a finitely generated module over $K\left[f_{0}, \ldots, f_{r}\right]$. Of course, this last $K$-algebra is isomorphic to the polynomial ring in $r+1$ variables.

Main Theorem Let $S, f_{0}, \ldots, f_{r}$ be as above. Then for every $g \in S$ such that

$$
f_{0} \cdot \in S \cdot f_{1}+\cdots+S \cdot f_{r},
$$

it follows

$$
g \in K[x] \cdot f_{1}+\cdots+K[x] \cdot f_{r}
$$

Remark This means that the relative Cohen-Macaulay property is satisfied. In the case when $S$ is an invariant subalgebra under some rational action by a reductive $K$-algabraic group on the polynomial ring, there exists the Reynholds operator. Therefore one can conclude that $g$ belongs to the ideal generated by $f_{1}, \ldots, f_{r}$. Using this, the relative result gives the Cohen-Macaulay property for invariant and graded subalgebras.

Perhaps it is the method of proof which is the most interesting part below. It consists of an ingenious reduction to characteristic $p$. It is elementary. 
Reduction to a small coefficient ring Let us first notice that it suffices to prove the theorem in the case when $g$ is a homogeneous polynomial. So this is assumed frow now on. Apart from $g, f_{0}, \ldots, f_{r}$ consider some finite set $h_{1}, \ldots, h_{m}$ in $S$ which generate $S$ as a module over $K\left[f_{\bullet}\right]$. There is also a finite set of polynomials $q_{1}, \ldots, q_{r}$ is $S$ such that

$$
f_{0} \cdot g=q_{1} \cdot f_{1}+\cdots+q_{r} \cdot f_{r} .
$$

The proof goes by a contradiction. Let us assume that $g$ does not belong to the ideal generated by $f_{1}, \ldots, f_{r}$ in $K[x]$. Let $N=\operatorname{deg}(g)$, while $d_{v}=\operatorname{deg}\left(f_{v}\right)$. Write

1. $g(x)=\sum c_{\alpha} x^{\alpha}$

2. $f_{v}(x)=\sum e_{v, \beta} x^{\beta}$.

To say that $g$ does not belong to the ideal generated by $f_{1}, \ldots, f_{r}$ in $K[x]$, means that the system of linear equations:

$$
c_{\alpha}=\sum_{\gamma, v} e_{\nu, \beta} \xi_{v, \gamma}
$$

can't be solved. Here $\xi$-variables appear when one tries to find an $r$-tuple of homogeneous polynomials

$$
\rho_{v}(x)=\sum_{\gamma \mid=N-d_{v}} \xi_{v, \gamma} \cdot x^{\gamma},
$$

such that $g=\rho_{1} \cdot f_{1}+\cdots+\rho_{r} \cdot f_{r}$. By Cramer's rule there exists a non-zero minor $\Delta$, expressed as a polynomial in the coefficients of $g, f_{1}, \ldots, f_{r}$, which by our present hypothesis rules out a solution to (2.1)

The ring $A \quad$ It is the $\mathbb{Z}$-algebra generated by coefficients of all the preceeding polynomials $g, f_{\bullet}, h_{\bullet}, q_{\bullet}$, together with the inverse of $\Delta$, i.e. the element $\Delta^{-1}$ in the field $K$.

We replace $K$ by $A$, and regard from now on the finitely generated ring over $A$ :

1. $S_{0}=\sum A\left[f_{0}, \ldots, f_{r}\right] \cdot h_{i}$

2. $\mathcal{R}_{0}=A\left[x_{1}, \ldots, x_{n}\right]$.

Notice that by the careful choice of $A$, we still have

$$
f_{0} \cdot g \in \sum_{v=1}^{v=r} S_{0} \cdot f_{v} .
$$

Next, by the generic flatness lemma (Matsumura 1980), there exists non-zero element $\beta$ in the ring $\mathcal{B}=A\left[f_{\bullet}\right]$, such that the localisation $\mathcal{R}_{0}\left[\beta^{-1}\right]$ is a free module over its subring $\mathcal{B}\left[\beta^{-1}\right]$.

Reduction to a finite field In the finitely generated $\mathbb{Z}$-algebra $A$ we pick a maximal ideal $\mathfrak{m}$, such that

$$
\Delta \in A \backslash \mathfrak{m} \text { and } \beta \in \mathcal{B} \backslash \mathfrak{m} \mathcal{B} \text {. }
$$

Since $A$ is a finitely generated $\mathbb{Z}$-algebra, it follows that $A / \mathfrak{m}$ is a finite field, denoted by $\bar{A}$.

Remark The last condition in (2.3), implies that $\bar{B}=\mathcal{B} / \mathfrak{m} \mathcal{B}$ is a subring of $\bar{A}[x]$. Moreover, since $f_{0}, \ldots, f_{r}$ from the start is algebraically independent, it follows that

$$
\bar{B}=\bar{A}\left[f_{0}, \ldots, f_{r}\right]
$$

is isomorphic to the polynomial ring $r+1$ variables over the field $\bar{A}$. This simple observation will be used below, when we introduce a certain free $\bar{B}$-module.

From the start we have:

$$
S_{0}=\mathcal{B}\left[h_{1}, \ldots, h_{m}\right]
$$


Denote by $\bar{h}_{v}$ the image in $\bar{A}$. Then :

$$
\overline{S_{0}}=\bar{B} \cdot \bar{h}_{1}+\cdots+\bar{B} \cdot \bar{h}_{m} .
$$

Passing to the quotient we also have:

$$
\overline{f_{0}} \cdot \bar{g}=\sum_{v=1}^{r} \bar{q}_{v} \cdot \bar{f}_{v},
$$

where $\bar{q}_{1}, \ldots, \bar{q}_{r}$ belong to $\bar{S}$. Next, choose a free $\bar{B}$-submodule $\mathcal{L}$ of finite rank in $\bar{S}$, such that the $\bar{B}$-module $S / \mathcal{L}$ has torsion. It gives some $\mathbf{c} \in \bar{B}$, such that

$$
\mathbf{c} \cdot \bar{S} \subset \mathcal{L} \text {. }
$$

Raising to high powers Let $p$ be the characteristic of the field $\bar{A}=A / \mathfrak{m}$. The field consists of $p^{N}$ elements and we set $q=p^{N}$.

Remark Recall the rule which says that when $\phi(x)$ and $\psi(x)$ is a pair of polynomials in $\bar{A}[x]$. Then one has:

$$
(\phi(x)+\psi(x))^{q}=\phi(x)^{q}+\psi(x)^{q} .
$$

Let $w$ be a posistive integer. Raising (2.4) above to the $w \cdot q$ th power, and multiplying with $\mathbf{c}$ give:

$$
\mathbf{c} \bar{g}^{w \cdot q} \cdot \bar{f}_{0}^{w \cdot q}=\sum_{v=1}^{r} \mathbf{c} \bar{q}_{v}^{w \cdot q} \cdot \bar{f}_{v}^{w \cdot q} .
$$

Next, for every positive integer $w$, it follows that the monomials $\left\{x^{\alpha}:|\alpha|<w \cdot q\right\}$, is a set of free generators when $\bar{A}[x]$ is regarded as a module over $\bar{A}\left[x^{w \cdot q}\right]$.

The choice of $w$ Choose $w$ so large that:

$$
\mathbf{c}=\sum_{|\alpha|<w \cdot q} c_{\alpha} \cdot x^{\alpha}
$$

An important observation Recall that $\left(f_{0}, f_{1}, \ldots, f_{r}\right)$ from the start were algebraically independent. Therefore they form a systeme of parameters in the ring $\bar{A}\left[f_{0}, f_{1}, \ldots, f_{r}\right]$. It follows that they also form a system of parameters in the free module $\mathcal{L}$

The final part of the proof The elements $\mathbf{c} \cdot \bar{g}$ and $\mathbf{c} \cdot \bar{q}_{i}$, belong to $\mathcal{L}$. Therefore (2.5) together with the important observation give an equation:

$$
\mathbf{c} \bar{g}^{w \cdot q}=\sum_{v=1}^{r} \bar{f}_{v}^{v \cdot q} \cdot h_{v}
$$

where $\left\{h_{v} \in \mathcal{L}\right\}$. Every polynomial $h_{v}(x)$ is expanded with respect to the basis of monomials, when $\bar{A}[x]$ as above is regarded as a free module over $\bar{A}\left[x^{w \cdot q}\right]$. Thus, we write:

$$
h_{v}(x)=\sum_{|\alpha|<w \cdot q} \rho_{v, \alpha} \cdot x^{\alpha} \cdot P_{v, \alpha}\left(x^{w \cdot q}\right) .
$$

Choose a non-zero coefficient $c_{\alpha}$ in the polynomial $\mathbf{c}(x)$. Then we get:

$$
c_{\alpha} \bar{g}^{w \cdot q} \in \sum \bar{A}\left[x^{w \cdot q}\right] \cdot \bar{f}_{v}^{w \cdot q} .
$$

In the finite field we recall that $\bar{A}^{1 / q}=\bar{A}$. Hence we can take the $\sqrt{w \cdot q}$-roots above, and obtain the inclusion:

$$
\bar{g} \in \sum \bar{A}[x] \cdot \bar{f}_{v} .
$$

But this is impossible! The reason is that the image of $\Delta$ in $\bar{A}$ is non-zero. Hence there exists a non-zero minor which rules out the solution to a linear system which would give the inclusion above, This contradiction finishes the whole proof. 


\section{Some Remarks}

We have announced the result for graded subalgebras of polynomials ring, since this gives the most common applications. However, the reader may observe that the sole essential rule was to find the minor $\Delta$. Since the decision to be an ideal over a polynomial ring is controlled by some sufficiently large degree, the main theorem remains true for every finitely generated $K$-subalgebra. Next, performing localizations over an affine and non-singular $K$-algebraic variety, one has a similar result, when the polynomial ring $K[x]$ is replaced by the $K$-algebra of regular functions over some non-singular affine variety. Of course, one may also pass to a sheaf-theoretic version, which also yields the relative Cohen-Macaulay property.

Question Can one extend the main theorem to the case when $K[x]$ is replaced by the $K$-algebra of regular functions on a Cohen-Macaulay variety?

The case of formal power series Replace the polynomials ring $K[x]$ by the local ring $O_{n}=K\left[\left[x_{1}, \ldots, x_{n}\right]\right]$ of formal power series. Here $S$ is assumed to be a finitely generated module over some $(r+1)$-tuple $f_{0}, \ldots, f_{r}$ which are formally independent, i.e. the formal completion of the ring they generate is isomorphic to $O_{r}$. In this situation the relative Cohen-Macaulay property still holds. To be precise, by the Artin-Rees lemma on can exhibit a minor $\Delta$ which yields an obstruction to the desired inclusion $g \in \sum O_{n} \cdot f_{v}$. A proof would go like this. Use first suitable primary decomposition and the normalisation theory (Björk 1979, chapter 3, paragraph 3.22 \& Nonkané 2013, section 3). Thereafter, perform divisions in such a way that the ideal generated by the $r$-tuple $f_{1}, \ldots, f_{r}$ is exhibited by Weierstrass polynomials. Then the proof starts as in the algebraic case, where the field $K$ will be some quotient field over the local ring of formal power series in the variables which do not appear in the Weierstrass polynomials. Using reductions in this field, and the Artin-Rees lemma would finish the proof.

Non-commutative versions Let us describe the relative Cohen-Macaulay theorem when one regards the Weyl algebra $A_{n}(K)$ over the field of characteristic zero. Consider a finitely generated commutative $K$-subalgebra $S$. In this case the main theorem extends as follows:

Start with the same hypothesis as in the main theorem the conclusion is then that $g$ belongs to the left ideal in $A_{n}(K)$, generated by $f_{1}, \ldots, f_{r}$.

A similar extension of the main theorem occurs if $S$ is a commutative subalgebra of an enveloping algebra $U(\mathfrak{g})$, where $\mathfrak{g}$ is a finite-dimensional Lie algebra.

To what extent a non-commutative extension of the previous theorem holds in unclear. But it would be interesting to investigate. For example, $\mathbb{C}$ the complex field. Consider some of the familiar $\mathbb{C}$-algebras of $A_{n}(\mathbb{C})$, such that the subalgebra $S$ of differential operators which are of Fuchsian type along an algebraic hypersurface, or more generally along some algebraic subvariety. There is always a "cheap extension" of the main theorem, where one, after a choice of some good filtration on $A_{n}(\mathbb{C})$, imposes the hypothesis that $g f_{0}$ belongs to the left ideal generated by some $r$-tuple $f_{1}, \ldots, f_{r}$ in the graded sense. Then one can begin to work with associated graded rings and reduce the whole proof to that of the main theorem.

Open problem The powerful result, and in particular the methods of proof from ( Hochster \& Huneke 1990) should be analyzed, in order to try to settle a very important question of great geometric significance. Namely, consider some complex analytic manifold $X$ and its cotangent bundle $T^{*}(X)$. Let $p$ be a point in $T^{*}(X)$ outside the zero section. Let $\mathcal{M}$ be a germ of a regular holonomic $\mathcal{E}_{X}$-module at $p$. Let $\Lambda$ be its support. Thus, $\Lambda$ is a germ of a conic lagrangian, such conic lagrangians are not arbitrary. This is understood via the micro-local version of the Riemann-Hilbert correspondence. The question, is to find out when $\Lambda$, regarded as an imbedded complex variety in the manifold $T^{*}(X)$, is Cohen-Macaulay. More generally, starting from the module $\mathcal{M}$, the question is whether it can be equipped with a good filtration such that $\operatorname{gr}(\mathcal{M})$ is Cohen-Macaulay.

\section{Acknowledgements}

The author would like to thank the anonymous referees for their comments and suggestions to improve the paper.

\section{References}

Atiyah, M. F., \& MacDonald, I. G. (1969). Introduction to commutative algebra. Addison Wesley, Reading MA.

Björk, J-E. (1979). Rings of differential operators. North-Holland Publishing Co., Amsterdam. 
Bruns, W., \& Herzog, J. (1998). Cohen-Macaulay rimngs. Cambridge University Press. https://doi.org/10.1017/CBO9780511608681.004

Cohen, I. S. (1946). On the structure and ideal theory of complete local rings. Transactions Amer. Math. Soc., 59, 54-106. https://doi.org/10.1090/S0002-9947-1946-0016094-3

Hochster, M., \& Huneke, C. (1990). Tight closure, invariant theory, and the Briançon Skoda theorem. J. Amer. Soc., 3, 31-116. https://doi.org/10.2307/1990984

Hochster, M., \& Roberts, J. (1974). Rings of invariants of reductive groups acting on regular rings are Cohen-Macaulay. Adv Math., 13, 31-116. https://doi.org/10.1016/0001-8708(74)90067-X

Knop, F. (1991). Die Cohen-Macaulay Eigenschaft von Invariantenringen. Unpublished.

Macaulay, F. S. (1916). The algebraic theory of modular systems. Cambridge Tracts in Mathematics and Mathematical Physics, 19. (Cambridge University Press). https://doi.org/10.3792/chmm/1263317740

Matsumura, H. (1980). Commutative algebra (2nd ed.). Reading: Benjamin.

Nonkané, I. (2013). The Weyl algebra and Noetherian oerators. Afr. Diaspora J. Math., 16(1), 59-69. https://projecteuclid.org/euclid.adjm/1391091307

Stanley, R. (1986). Combinatorics and Commutative Algebras. Second edition. Birkhauser Press, Boston, MA.

\section{Copyrights}

Copyright for this article is retained by the author(s), with first publication rights granted to the journal.

This is an open-access article distributed under the terms and conditions of the Creative Commons Attribution license (http://creativecommons.org/licenses/by/4.0/). 